\title{
Prognostic Biomarkers in Melanoma: The Legacy of a Surgeon Scientist Who Followed the Data
}

\author{
Giorgos Karakousis, $\mathrm{MD}^{1}$, and Jonathan Zager, $\mathrm{MD}^{2}$ \\ ${ }^{1}$ Hospital of the University of Pennsylvania, Philadelphia, PA; ${ }^{2} \mathrm{H}$ Lee Moffitt Cancer Center, Tampa, FL
}

The significance of the local immune environment of primary melanoma remains elusive. Specifically, the prognostic significance of histopathologic features such as tumor-infiltrating lymphocytes (TILs) or regression have been variably reported in the literature with controversial results. In the original Clark model of prognostic factors in early-stage (stage I) melanoma published in 1989, TIL was one of six primary tumor factors significantly associated with long-term survival. ${ }^{1}$ After the advent of sentinel lymph node (SLN) biopsy a few years later, the independent prognostic significance of TIL subsequently came into question, with several studies finding TIL to be inversely associated with SLN metastasis, but not disease-specific survival, when SLN status was accounted for in prognostic models. The story of regression is even more complex, with several studies finding a positive association with improved survival and many others finding a negative correlation. Moreover, the association of regression with SLN status has not been well-characterized to date, with some studies suggesting no association of this histopathologic feature with SLN metastasis and others finding a positive correlation, perhaps by virtue of regression, leading to an underestimation of the true thickness of the primary melanoma. These controversies, which may in part result from inconsistency in pathologic assessment of these histopathologic variables, more importantly underscore the

(C) Society of Surgical Oncology 2021

First Received: 13 December 2021

Accepted: 15 December 2021;

Published Online: 2 January 2022

G. Karakousis, MD

e-mail: giorgos.karakousis@uphs.upenn.edu need to gain a deeper molecular understanding into the local immune microenvironment that may not be captured with conventional histopathology alone.

In this issue of Annals of Surgical Oncology, Dr. Han and colleagues study the relationship of TIL and regression in 2450 patients with primary melanoma using a large, multicenter, international database through the Sentinel Lymph Node Working Group. ${ }^{2}$ Notably, they found TIL to be strongly associated with regression and inversely associated with SLN metastasis (the latter driven predominantly by brisk TILs). Interestingly, among SLNpositive patients, the presence of TIL was not statistically associated with improved overall survival (OS). While regression was not associated with SLN metastasis or OS, when combined with TIL it appeared to augment the prognostic significance of TIL, particularly when compared with the absence of both histopathologic features. This suggests the possibility of an important interaction between these two pathologic variables, and their co-existence (or absence) may signify distinct prognostically relevant immune response phenotypes reflecting diverse local immune microenvironments. The authors should be highly commended for using large datasets in a way that can sort out these nuanced interactions and bring greater understanding in a controversial area.

This study is but one of many published by Dr. Dale Han and colleagues that sought to gain a greater understanding of prognostic biomarkers in melanoma using large datasets. Dr. Han was born on 16 June 1976 in New York and passed away recently and unexpectedly on 2 October 2021 in Bethany, Oregon (Fig. 1). He received his undergraduate and medical school degrees at the State University of New York at Stony Brook. He went on to complete his general surgery residency training at the Hospital of the University of Pennsylvania, prior to going on to complete his Complex General Surgical Oncology fellowship at the 


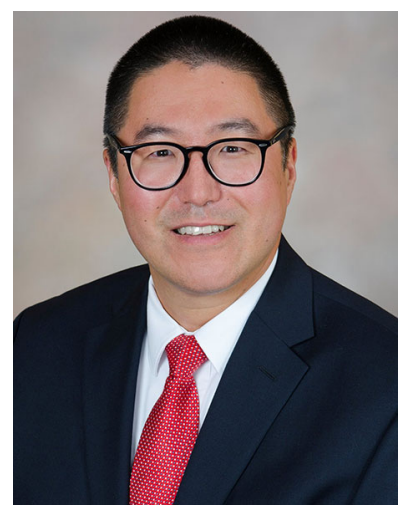

FIG. 1 Dr. Dale Han (1976-2021)

H Moffitt Cancer Center in Tampa, Florida. Dr. Han was one of the first in the country to be board-certified in Complex General Surgical Oncology after fellowship and then took a faculty position at Yale University, where he quickly gained national recognition in the field of melanoma through multiple publications and presentations. In 2018, he was recruited to Oregon Health \& Science University as an Associate Professor, where he continued to take care of multitudes of grateful patients with melanoma and other cutaneous and soft tissue malignancies, and continued his research in melanoma, with a specific interest in prognostic biomarkers. He remained very active in the
Society of Surgical Oncology and was a member of the Melanoma Disease Site Working Group and also served as the Chair of the Scientific Committee of the Sentinel Lymph Node Working Group.

Dr. Dale Han was a kind, compassionate surgeon who deeply cared about his patients and was devoted to his family. His passion for research was driven by a desire to better help his patients, and he steadfastly listened to the data without holding fast to preconceived notions. His relentless approach to scientific discovery was matched by his passion for mentorship and educating the next generation of surgeons. He embodied the very best of qualities and values we strive for as surgical oncologists. We will deeply miss, but never forget, our colleague, but most notably, our friend, who will continue to inspire us.

\section{REFERENCES}

1. Clark WH Jr, Elder DE, et al. Model predicting survival in stage I melanoma based on tumor progression. J Natl Cancer Inst. 1989;81(24):1893-904.

2. Morrison S, Han G, et al. Is there a relationship between TIL and regression in melanoma? Ann Surg Oncol 2021. https://doi.org/10. 1245/s10434-021-11251-z.

Publisher's Note Springer Nature remains neutral with regard to jurisdictional claims in published maps and institutional affiliations. 